\title{
Brittle Cylinder Transfering by a Manipulator with Three Fingers
}

\author{
Yury F. Golubev ${ }^{1,2}$, Elena V. Melkumova ${ }^{2}$ \\ ${ }^{1}$ Keldysh Institute of Applied Mathematics, RAS, Moscow, 125047, Russia \\ ${ }^{2}$ M.V. Lomonosov State University, Moscow, 119991 Russia \\ elena_v_m@mail.ru
}

\begin{abstract}
We consider the problem of brittle cylinder grasping by the manipulator fingers. Each finger contacts the cylinder in a single supporting point. Using numerical simulations and analytically, possible locations of the contact points on the cylinder, for which there is a kinetostatics problem solution when the cylinder is moved by three fingers, are received. There is an analogy of the equilibrium of a three-legged robot on a cylinder for the problem of transfer by a manipulator with a three-finger grasp of a cylinder.
\end{abstract}

Keywords: three-finger grasp, Amontons-Coulomb friction, three-legged robot.
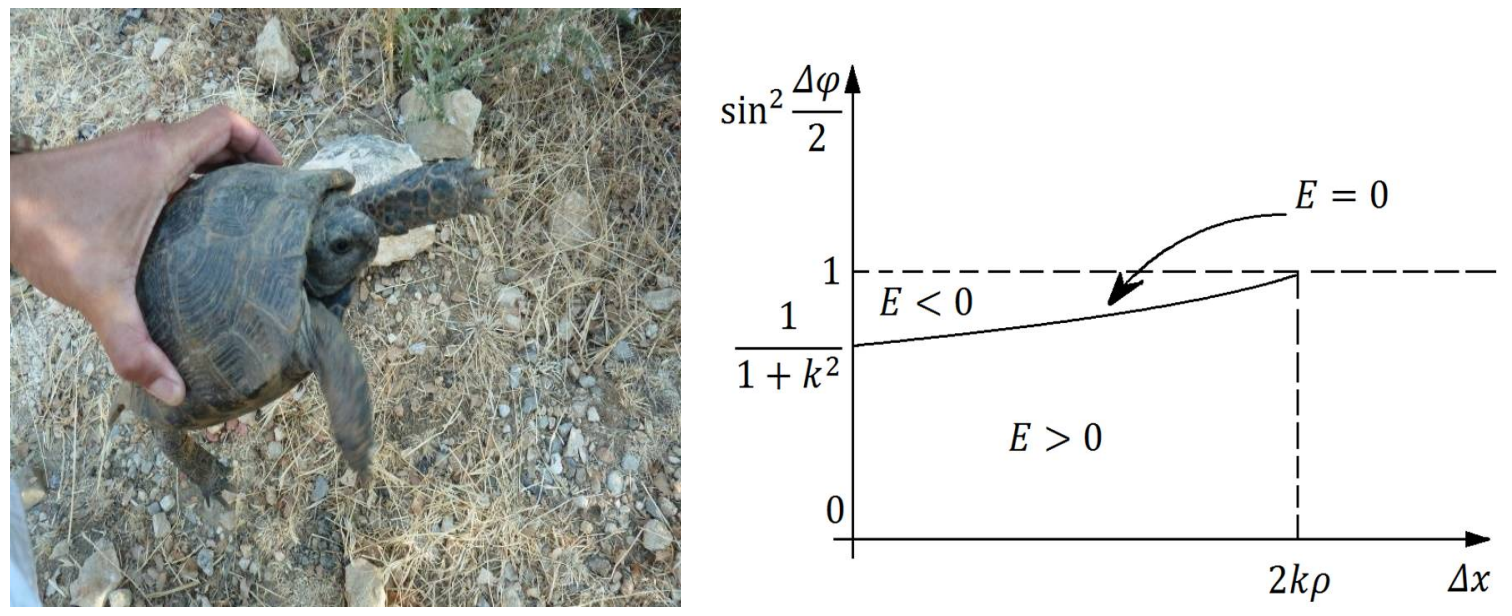

If during driving the car you see the turtle on the road, you save it. But how one robot with a manipulator can help to another. Let the corpus is cylinder. How to hold it. The analytical and the numerical parameter diagrams for two fields of supporting points are shown.

\section{Possible configurations are shown in the plot}

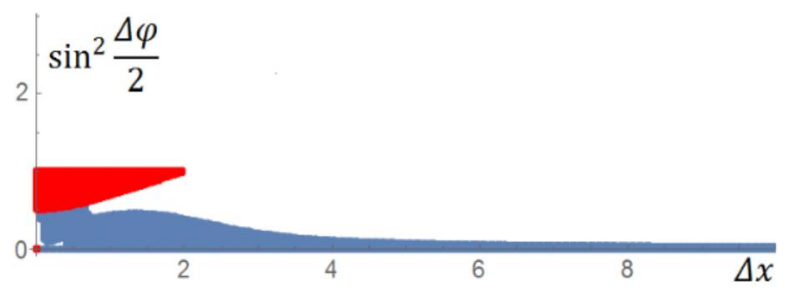

$$
\begin{gathered}
\alpha=\pi / 4, \\
x_{2}=-x_{1}, \varphi_{2}=-\varphi_{1}
\end{gathered}
$$




\title{
Перенос хрупкого цилиндра манипулятором с трёхпальцевым схватом
}

\author{
Ю.Ф. Голубев ${ }^{1}$, Е.В. Мелкумова ${ }^{2}$ \\ ${ }^{1}$ ИПМ имени М.В. Келдыша РАН, Россия, Москва, 125047, Миусская пл., д. 4 \\ ${ }^{2}$ МГУ имени М.В. Ломоносова, Россия, Москва, 119991, ГСП-1, Ленинские горы, д. 1 \\ elena_v_m@mail.ru
}

\begin{abstract}
Аннотация
Исследуется задача об удержании хрупкого прямого кругового шероховатого цилиндра пальцами рук робота манипулятора. Каждый из пальцев имеет одну точку в контакте с цилиндром. Численно и аналитически получены возможные области расположения точек контакта на цилиндре, для которых существует решение задачи кинетостатики при переносе цилиндра двумя и тремя пальцами. Эта задача имеет аналогии задачам о равновесии многоногого шагающего робота на поверхности объекта цилиндрической формы или робота, опирающегося на произвольную поверхность, точки подвеса ног которого на корпусе расположены на поверхности цилиндра.
\end{abstract}

Ключевые слова: трехпальцевый манипулятор, шагающий робот, цилиндр.

\section{1. Введение}

Исследуется задача об удержании хрупкого цилиндра пальцами манипулятора [1]. Эта задача актуальна, например, при опрокидывании одного шагающего робота с цилиндрическим корпусом, тогда как другой помогает ему подняться. В природе известны примеры взаимопомощи у черепах. Каждый из пальцев робота имеет одну точку в контакте с цилиндром. Численно и аналитически получены возможные области расположения точек контакта на цилиндре, для которых существует решение задачи кинетостатики при переносе цилиндра тремя пальцами.

Задача о захвате объекта произвольной формы, например прямого кругового цилиндра, аналогична задаче о существовании заданного движения шагающего робота, состоящего из корпуса, $l$ рук с $m$ пальцами и $n$ ног, опирающегося о хрупкий шероховатый цилиндр, достаточного радиуса $\rho$, в $n+m$ точках опоры. Предполагается, что руки, пальцы и ноги робота совершают заданное движение относительно корпуса робота.

Эта работа возникла, как развитие аналогичных исследований для перемещения шагающего аппарата по горизонтальной плоскости. Первый шаг в исследовании сходной проблемы был сделан Н.Е. Жуковским. В работе [1] он исследовал равновесие твёрдого тела на плоскости при наличии трения.

Движение шестиногого шагающего аппарата в режиме квазистатики по плоскости исследовано в работе Д.Е. Охоцимского и Ю.Ф. Голубева [2] при этом использован следующий критерий статической устойчивости шагающего робота. Шагающий робот, опирающийся о горизонтальную плоскость, статически устойчив в некоторый момент времени тогда и только тогда, когда вертикальная проекция его центра масс на опорную поверхность лежит строго внутри опорного многоугольника, соответствующего этому моменту. Возникает естественный вопрос, как выглядят аналогичные критерии для других поверхностей. В том числе критерий неопрокидывания робота. В настоящей работе изучена аналогия этой задачи - захват объекта пальцами руки манипулятора. Численно и аналитически получены области существования решения задачи о распределении реакций, для шагающего робота и для схвата, 
изучена их структура. Модельные задачи динамики и управления двуногой ходьбой на плоскости изучены В.В. Белецким в [3].

\section{2. Постановка задачи}

Рука человека имеет пять пальцев. В работе [4] показано, что при увеличении числа точек опоры шагающего робота внутри гладкого горизонтального цилиндра до трех, опорное множество разбивается на два подмножества. Аналогично на рис. 1 показан захват панциря черепахи рукой человека. По одну сторону один палец, а по другую четыре. Фактически хватило бы и двух. Однако для более комфортного и уверенного захвата желательно использовать три пальца. Для более сложных поверхностей или при изменении точек захвата не лишними, как показывает практика все пять пальцев руки.

Предполагается, что руки, пальцы и ноги робота совершают заданное движение относительно корпуса робота. Рассматривается кинетический момент робота относительно точки O. Для того, чтобы заданное движение могло быть реализовано, реакции $\tilde{\mathbf{R}}_{i}$ в $n$ точках опоры должны удовлетворять следующим уравнениям [1] кинетостатики:

$$
\sum_{i} \tilde{\mathbf{R}}_{i}=-\tilde{\boldsymbol{\Phi}}, \quad \sum_{i} \tilde{\mathbf{r}}_{i} \times \tilde{\mathbf{R}}_{i}=-\tilde{\mathbf{M}}, \quad i=1, \ldots, n,
$$

где $\tilde{\mathbf{R}}_{i}$ - компоненты реакций; $\tilde{\mathbf{r}}_{i}$ - радиус-векторы точек опоры; $\tilde{\boldsymbol{\Phi}}$ - сумма даламберовых сил инерции и внешних активных сил; $\tilde{\mathbf{M}}$ - сумма моментов даламберовых сил инерции и моментов внешних активных сил относительно неподвижной точки $O$. Первое векторное уравнение отвечает за изменение количества движения робота. Оно эквивалентно трём скалярным уравнениям в проекции на выбранные оси. Второе уравнение системы (1) определяет заданное изменение кинетического момента.
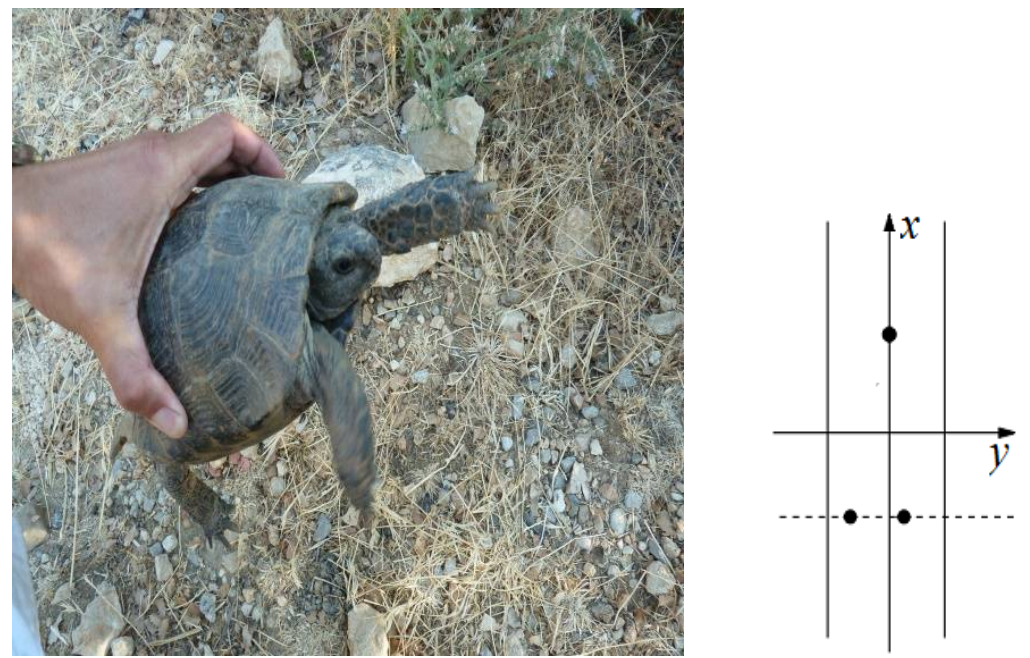

Рис. 1. Разделение опорного множества на два подмножества.

Ограничимся случаем, когда $\tilde{\boldsymbol{\Phi}} \neq 0$ и перпендикулярно $\tilde{\mathbf{M}}$. Приведем систему $\{\tilde{\boldsymbol{\Phi}}, \tilde{\mathbf{M}}\}$ к равнодействующей в точке $C$ [2]

$$
\tilde{\mathbf{r}}_{C} \times \tilde{\boldsymbol{\Phi}}=\tilde{\mathbf{M}}, \quad \mathbf{r}_{C}=-\frac{\tilde{\mathbf{M}} \times \tilde{\boldsymbol{\Phi}}}{\tilde{\boldsymbol{\Phi}}^{2}}
$$

где $\tilde{\mathbf{r}}_{C}$ - радиус-вектор точки $C$. Точка $C$ будет также точкой приложения равнодействующей реакций. Например, точка $C$ может быть центром масс переносимого объекта. 
Далее рассматривается задача о распределении реакций $\tilde{\mathbf{R}}_{i}$ по точкам опоры в некоторый фиксированный момент времени в предположении, что сила $\tilde{\mathbf{\Phi}}$ приложена в точке $C$, а силовой момент в этой точке отсутствует.

Уравнения (1) для нахождения реакций при заданном движении корпуса и ног робота могут быть представлены в виде

$$
\sum_{i} \tilde{\mathbf{R}}_{i}=-\tilde{\boldsymbol{\Phi}}, \quad \sum_{i} \tilde{\mathbf{r}}_{i} \times \tilde{\mathbf{R}}_{i}=-\tilde{\mathbf{r}}_{C} \times \tilde{\boldsymbol{\Phi}}
$$

В том случае, когда поверхность реализует неудерживающую связь, имеются дополнительные ограничения на направления нормальных составляющих $\tilde{N}_{i}$ реакций $[1,3]$ : $\tilde{N}_{i}=\tilde{\mathbf{R}}_{i} \cdot \mathbf{e}_{i}^{v} \geq 0$, где $\mathbf{e}_{i}^{v}-$ внешняя нормаль к поверхности цилиндра в $i$-ой точке опоры.

Касательные компоненты реакций представим в виде $\tilde{\mathbf{F}}_{i}=\tilde{\mathbf{R}}_{i}-\tilde{N}_{i} \mathbf{e}_{i}^{v}$. Уравнения (2) дополняются неравенствами, отвечающими за попадание реакций в соответствующие конусы трения: $\left|\tilde{\mathbf{F}}_{i}\right| \leq k \tilde{N}_{i}, i=\overline{1, n}$.

Хрупкость цилиндра означает выполнение неравенств

$$
\tilde{N}_{i} \leq l, i=\overline{1, n}
$$

Задача о распределении (существовании) реакций формулируется следующим образом. Найти реакции $\tilde{\mathbf{R}}_{i}$, удовлетворяющие следующей системе уравнений и неравенств:

$$
\begin{gathered}
\sum_{i} \tilde{\mathbf{R}}_{i}=\tilde{\boldsymbol{\Phi}}, \quad \sum_{i} \tilde{\mathbf{r}}_{i} \times \tilde{\mathbf{R}}_{i}=\tilde{\mathbf{r}}_{c} \times \tilde{\boldsymbol{\Phi}}, \\
\tilde{N}_{i} \geq 0,\left|\tilde{\mathbf{F}}_{i}\right| \leq k \tilde{N}_{i}, i=\overline{1, n}
\end{gathered}
$$

Коэффициент сухого трения $k$ будем считать одинаковым для всех точек опоры.

Первое неравенство (4) есть следствие того, что цилиндр - неудерживающая поверхность, второе - условие того, что касательные компоненты реакций $\tilde{\mathbf{F}}_{i}$ ограничены предельным значением кулоновского сухого трения. Как только $\left|\tilde{\mathbf{F}}_{i}\right|$ превышает предельное значение, возникает проскальзывание ноги относительно поверхности.

Если решение задачи (3), (4) существует, то требуемое движение реализуемо. В противоположном случае - не реализуемо.

Задача о рациональном распределении или выборе реакций опоры возникает в связи с наличием статической неопределённости: при заданной кинематике движения реакции в точках опоры определены зачастую неоднозначно. Они образуют некоторое множество, отсюда возникает возможность учёта дополнительных требований, выполнение которых желательно в процессе передвижения.

Пусть точки опоры робота расположены на поверхности шероховатого прямого кругового цилиндра с коэффициентом $k$ сухого трения, рис. 2. Правоориентированные декартовы оси $O x y z$ свяжем с цилиндром (рис. 2). Ось $O x$ пустим по оси цилиндра так, чтобы проекция $\tilde{\boldsymbol{\Phi}}$ на эту ось была отрицательной. Ось $O z$ введём так, чтобы плоскость $O x z$ была параллельна вектору $\tilde{\boldsymbol{\Phi}}$. Пусть ось цилиндра составляет угол $\alpha$ с вектором $\tilde{\boldsymbol{\Phi}}$. Задача о существовании реакций (3), (4) имеет прямую аналогию с задачей о существовании реакций в однородном поле силы тяжести, когда упомянутые точки опоры расположены на внешней поверхности шероховатого наклонного прямого кругового цилиндра, ось симметрии которого расположена под углом $\alpha$ с вектором вертикали. Работа продолжает исследование [4], где была рассмотрена проблема поиска решения задачи о распределении реакций при $\alpha=0$ и работ $[5,6]$ - при произвольном $\alpha$.

В системе $O x y z$ представим координаты реакции поверхности в $i$-ой точке опоры, силы $\tilde{\boldsymbol{\Phi}}$, точки $C$ приложения равнодействующей активных сил и сил инерции соответственно 


$$
\tilde{\mathbf{R}}_{i}=\left(\tilde{R}_{i}^{x}, \tilde{R}_{i}^{y}, \tilde{R}_{i}^{z}\right), \tilde{\mathbf{\Phi}}=(-\tilde{\Phi} \sin \alpha, 0,-\tilde{\Phi} \cos \alpha), \tilde{\mathbf{r}}_{C}=\left(\tilde{x}_{c}, \tilde{y}_{c}, \tilde{z}_{c}\right),
$$

где $\tilde{\Phi}$ - модуль активной силы приложенной к роботу.
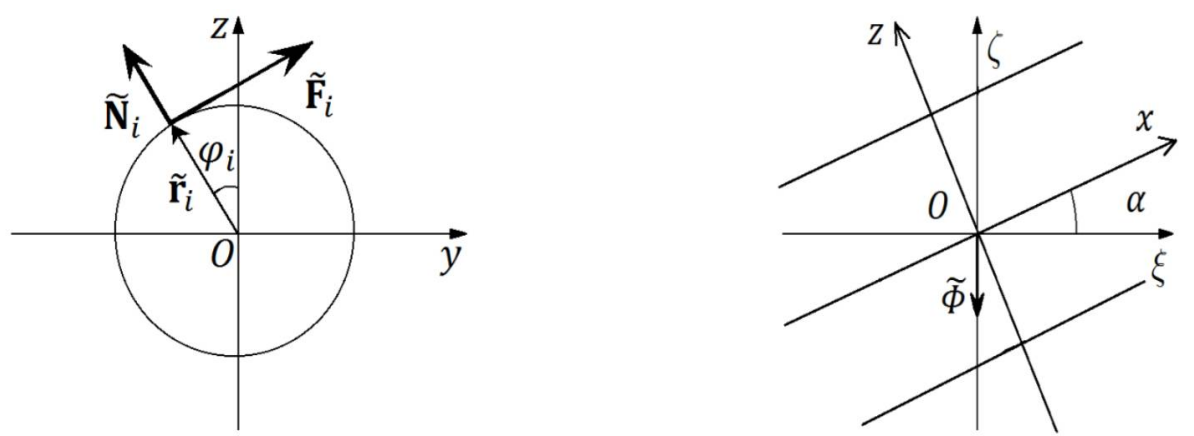

Рис. 2. Хрупкий цилиндр

В системе координат $O x y z$, используя цилиндрические координаты, зададим радиусвектор, вектор внешней нормали к поверхности цилиндра, нормальную составляющую реакции в $i$-ой точке опоры робота соответственно:

$$
\begin{gathered}
\tilde{\mathbf{r}}_{i}=\left(\tilde{x}_{i},-\rho \sin \varphi_{i}, \rho \cos \varphi_{i}\right), \quad \mathbf{e}_{i}^{v}=\left(0,-\sin \varphi_{i}, \cos \varphi_{i}\right), \\
\tilde{\mathbf{N}}_{i}=\left(0,-\tilde{N}_{i} \sin \varphi_{i}, \tilde{N}_{i} \cos \varphi_{i}\right),
\end{gathered}
$$

где $\rho$ - радиус цилиндра; $\varphi_{i}$ - угол между осью $O z$ и вектором $\mathbf{e}_{i}^{v}$.

Обозначим через $\mathbf{e}_{x}$ единичный вектор оси $O x$. Зададим единичный вектор касательной к основанию цилиндра: $\mathbf{e}_{i}^{\tau}=\left(0, \cos \varphi_{i}, \sin \varphi_{i}\right)$. Тогда касательная составляющая реакции в $i$-ой точке опоры в системе координат Охуz примет вид: $\tilde{\mathbf{F}}_{i}=\left(\tilde{F}_{i}^{x}, \tilde{F}_{i}^{y z} \cos \varphi_{i}, \tilde{F}_{i}^{y z} \sin \varphi_{i}\right)$, где $\tilde{F}_{i}^{x}=\tilde{\mathbf{F}}_{i} \cdot \mathbf{e}_{x}, \tilde{F}_{i}^{y z}=\tilde{\mathbf{F}}_{i} \cdot \mathbf{e}_{i}^{\tau}$, продольная и касательная к основанию цилиндра составляющие касательной компоненты реакции в $i$-ой точке опоры.

После преобразований из двух векторных уравнений (3) получим шесть скалярных. Это система уравнений кинетостатики

$$
\begin{gathered}
\sum_{i=1}^{n}\left(F_{i}^{x}\right)=\sin \alpha, \\
\sum_{i=1}^{n}\left(-N_{i} \sin \varphi_{i}+F_{i}^{y z} \cos \varphi_{i}\right)=0, \\
\sum_{i=1}^{n}\left(N_{i} \cos \varphi_{i}+F_{i}^{y z} \sin \varphi_{i}\right)=\cos \alpha, \\
\sum_{i=1}^{n}\left(-F_{i}^{y z}\right)=y_{c} \cos \alpha, \\
\sum_{i=1}^{n}\left(-x_{i} N_{i} \cos \varphi_{i}-x_{i} F_{i}^{y z} \sin \varphi_{i}+F_{i}^{x} \cos \varphi_{i}\right)=z_{c} \sin \alpha-x_{c} \cos \alpha, \\
\sum_{i=1}^{n}\left(-x_{i} N_{i} \sin \varphi_{i}+x_{i} F_{i}^{y z} \cos \varphi_{i}+F_{i}^{x} \sin \varphi_{i}\right)=-y_{c} \sin \alpha,
\end{gathered}
$$


где $N_{i}=\frac{\tilde{N}_{i}}{\tilde{\Phi}}, F_{i}^{x}=\frac{\tilde{F}_{i}^{x}}{\tilde{\Phi}}, F_{i}^{y z}=\frac{\tilde{F}_{i}^{y z}}{\tilde{\Phi}}, x_{c}=\frac{\tilde{x}_{c}}{\rho}, y_{c}=\frac{\tilde{y}_{c}}{\rho}, z_{c}=\frac{\tilde{z}_{c}}{\rho}, x_{i}=\frac{\tilde{x}_{i}}{\rho}$.

В случае двух точек опоры модель дополняется введением момента трения верчения в точках контакта. Для трёх точек опоры ограничимся рассмотрением сухого трения. Результаты распространяются на случай скольжения точек опоры с трением Амонтона-Кулона.

Уравнения кинетостатики дополняются неравенствами, отвечающими за попадание реакций в соответствующие конусы трения [2]. Первая группа неравенств есть следствие того, что цилиндр - неудерживающая поверхность. При внутреннем захвате объекта скалярное произведение реакции на главную нормаль к поверхности в точке опоры должно быть неотрицательно: $\tilde{N}_{i}=\tilde{\mathbf{R}}_{i} \cdot \mathbf{e}_{i}^{v} \geq 0$, где $\mathbf{e}_{i}^{v}-$ внешняя нормаль к поверхности цилиндра в $i$-ой точке опоры. При внешнем захвате, указанные неравенства имеют обратный знак. Хрупкость цилиндра означает ограничение на модуль нормальных составляющих реакций.

Если число точек контакта $n+m$ чётно, и они попарно расположены в совпадающих точках по разные стороны поверхности практически нулевой толщины, то указанные неравенства выполняются.
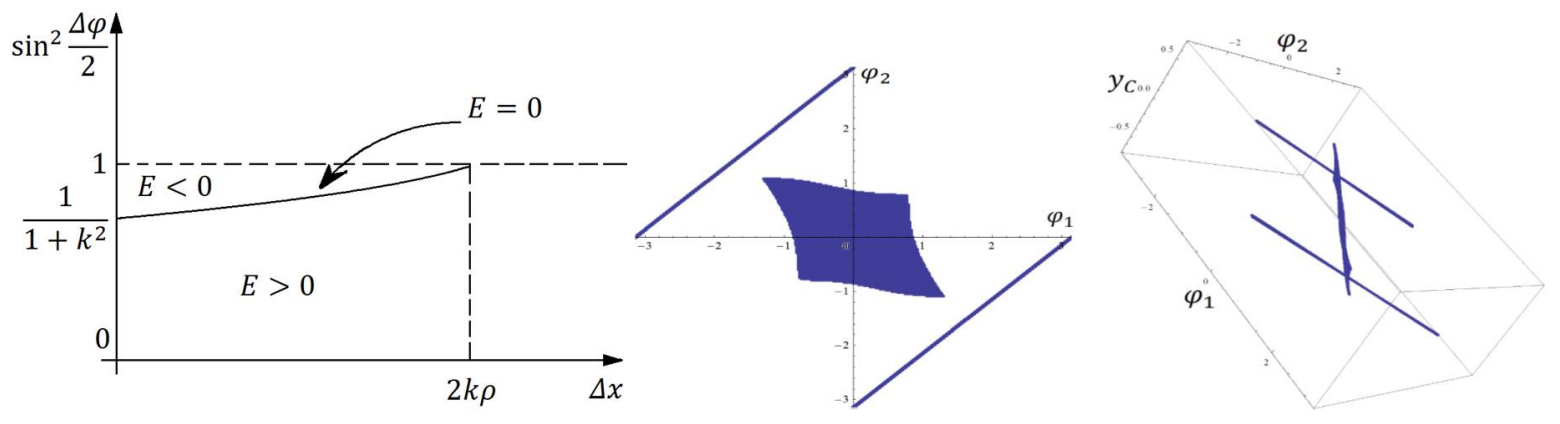

Рис. 3. Области параметров, для которых задача о распределении реакций имеет решение

Вторая группа неравенств это условие того, что касательные компоненты реакций ограничены предельным значением кулоновского сухого трения. То есть уравнения кинетостатики [3] дополняются квадратичными неравенствами, отвечающими за попадание реакций в соответствующие конусы трения. Как только модули касательных реакций превышают предельное значение, возникает проскальзывание ноги или пальца относительно поверхности. Это квадратичные неравенства, относительно параметра $p$, где $p$ - разность компонент реакций вдоль оси цилиндра, $E$ совпадающий и не зависящий от $\alpha$ коэффициент при $p$ в квадрате. Заметим, что для произвольной поверхности структура и свойства этих неравенств сохраняются, рис. 3 .

При движении робота происходит смена одноопорной, двухопорной [4] и трёхопорной фаз. Аналогично рука манипулятора осуществляет захват двумя или тремя пальцами. Жонглировать может и одним, когда центр масс над точкой опоры.

Пусть $n=1$. Искомое условие существования движения состоит в том, что реакция в единственной точке опоры должна быть равна силе $\tilde{\boldsymbol{\Phi}}$ по модулю, а опорная точка расположена на прямой вдоль силы $\tilde{\boldsymbol{\Phi}}$, проходящей через точку $C$. При этом, наклон линии действия силы $\tilde{\boldsymbol{\Phi}}$ к нормали не должен превышать угла трения.

На рис. 2 показаны оси, связанные с цилиндром, углы отклонения точек опоры от оси, связанной с цилиндром, угол наклона оси цилиндра, например, к горизонту. Для двуопорной фазы рассмотрены конфигурации робота симметричные относительно точки $C$ вдоль, и поперек оси цилиндра. При $x_{2}=-x_{1}, \varphi_{2}=-\varphi_{1}$ для углов $\alpha=0$ и $\alpha=\pi / 4$, ниже, рис. 4 , приведены графики, для областей $E<0$ и $E \geq 0$. Эти графики дополняют и суммируют полученные результаты [5], [6]. 


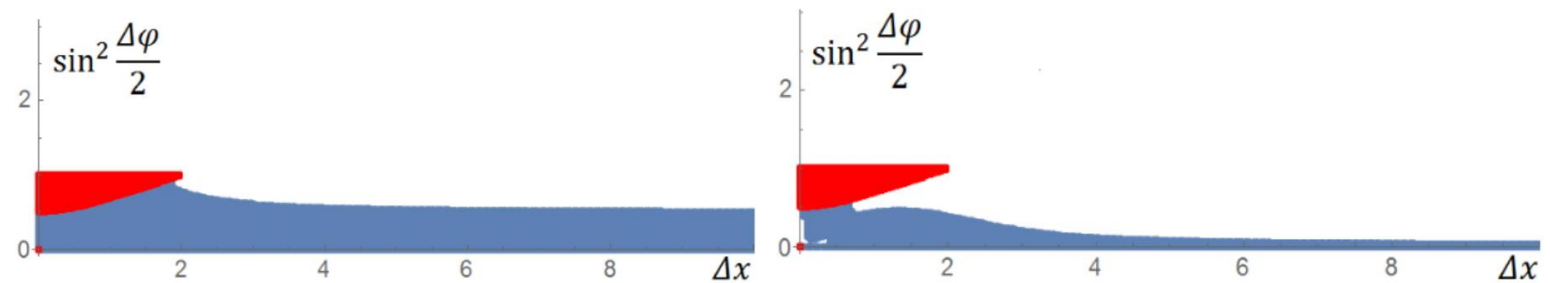

Рис. 4. Симметричные вдоль оси цилиндра конфигурации робота

Для конфигураций пальцев робота, симметричных относительно точки $C$ вдоль оси цилиндра, рассмотрены три случая с неотрицательным коэффициентом $E$, для расстояния $x$, между точкой $C$ и опорными точками: $0.9,1$ и 1.1 при $\rho$ и $k$ равным $1, \alpha$ от 0 до $\pi$ (всего 13 различных величин угла наклона цилиндра) [7], [8].

На рис. 1 рука человека удерживает корпус черепахи расположенный под углом $\pi / 4$ к горизонту. Построены области существования решения задачи о распределении реакций на плоскости двух углов, отвечающих проекциям точек опоры на основание цилиндра и трёхмерные области, дополняющие указанную плоскость аппликатой точки $C$. При $\alpha: 0, x: 1$, область состоит из трёх отдельно расположенных связных подобластей. На плоскости углов каждая из пары параллельных прямых соответствует опоре в диаметральной плоскости цилиндра содержащей точку $C$.

Между этими прямыми расположена связная область. Она содержит отрезок прямой, соответствующий равенству углов, робот опирается сверху, на прямую, параллельную оси цилиндра, удовлетворяющей ограничению на отклонения от направления силы. Указанный отрезок на графиках исчезает при $x=0.9$ для $\alpha$ равном $\pi / 4$, а при увеличении $x$, позже, при $4 \pi / 9$ [9], [10]. Это соответствует тому, что цилиндр начинает скользить в схвате робота с двумя точками опоры. Поэтому третий палец оказывается не лишним. Допустимые области для $\alpha=\pi / 3 ; x=1.1$ построены в работах [11]-[14]. Результаты полученные в [4], [5] для шагающего робота с тремя точками опоры имеют аналогию для схвата с тремя точками опоры удерживающего цилиндр.

На рис. 1 показан случай трёх точек в опоре и слева одна из возможных опорных троек. Большой палец руки оказывается на одном конце диаметра цилиндрического панциря. Остальные пальцы находятся в окрестности решения для гладкого цилиндра, когда две точки опоры расположены на одной прямой ортогональной оси цилиндра.

Переворачивание шагающего робота в рабочее состояние из аварийного положения “вверх ногами” рассмотрено в работе [15]. Черепаха, например, переворачивается путем отжимания от поверхности. Альтернативным методом может быть помощь другого шагающего робота с трехпальцевым манипулятором. Если конечно робот не в силах выполнить трюк волчка тип-топ.

\section{4. Заключение}

Робот может удерживать объект с произвольной поверхностью, например, цилиндр, одним пальцем с внутренней или внешней стороны, так, что центр масс поверхности располагается, соответственно под или над точкой контакта. При этом, наклон линии действия силы $\tilde{\boldsymbol{\Phi}}$ к нормали не должен превышать угла трения.

Схват может удерживать цилиндр двумя пальцами, расположенными на одном диаметре. И несколькими парами пальцев, расположенных по разные стороны от тонкой поверхности объекта в совпадающих точках.

Если цилиндр хрупкий, то двух пальцев может оказаться недостаточно. Робот может удерживать горизонтальный хрупкий цилиндр тремя пальцами, расположенными на внешней поверхности нижнего горизонтального полуцилиндра. При этом нагрузка на три пальца 
распределяется более равномерно, так что величины нормальных реакций не превышают заданной величины $l$. Например, пусть одна из точек опоры и центр масс цилиндра расположены в вертикальной плоскости содержащей ось цилиндра, а две другие точки опоры в плоскости ортогональной оси цилиндра при этом центр масс цилиндра расположен внутри опорного треугольника. Аналогично, если схват держит цилиндр на его внутренней поверхности, то три точки опоры должны быть расположены на верхнем полуцилиндре. Три точки опоры схвата могут быть расположены в вертикальной плоскости, содержащей ось цилиндра. Также они могут быть расположены в вертикальном сечении цилиндра.

\section{Благодарности и ссылки на гранты} руки.

Выражаем благодарность коллеге, продемонстрировавшему захват черепахи пальцами Работа выполнена при финансовой поддержке РФФИ (проект № 19-01-00123 А).

\section{Литература}

1. Жуковский Н.Е. Условие равновесия твёрдого тела, опирающегося на неподвижную плоскость некоторой площадкой и могущего перемещаться вдоль этой плоскости с трением. Полное собрание сочинений, Т. 1, - М.-Л.: ОНТИ НКТП СССР главная редакция авиационной литературы, 1937, С. 433-449.

2. Охоцимский Д.Е., Голубев Ю.Ф. Механика и управление движением автоматического шагающего аппарата. М.: Наука, 1984. 312 с.

3. Голубев Ю.Ф. Основы теоретической механики. М.: МГУ, 2019. 728 с.

4. Голубев Ю.Ф., Мелкумова Е.В. Условия статической устойчивости шагающего аппарата в горизонтальном цилиндре и на двух плоскостях // Известия Российской академии наук. Теория и системы управления. 1999. № 2. С. 116-122.

5. Мелкумова Е.В. Колебания шагающего аппарата вокруг оси гладкого опорного цилиндра// Вестник Московского университета. Серия 1: Математика. Механика. 2000. № 5. С. 47-49.

6. Голубев Ю.Ф., Мелкумова Е.В. Равновесие двуногого робота на шероховатом горизонтальном цилиндре с учётом реакций вдоль его оси. М.: МАКС Пресс, 2010. 64 с.

7. Golubev Yu.F., Melkumova E.V. Walking Robot Dynamics on a Rough Inclined Cylinder.//ESMC2012 - 8th European Solid Mechanics Conference. Graz, Austria, July 9-13, 2012. Book of Abstracts. Ed. Holzapfel G.A. and Ogden R.W.

8. Golubev Yu.F., Melkumova E.V. Prescribed Motion of a Two-legged Walking Robot on a Rough Cylinder. Proceedings of 2016 International Conference "Stability and Oscillations of Nonlinear Control Systems" (Pyatnitskiy's Conference), Moscow, Trapeznikov V.A. Institute of Control Sciences, pp. 1-4.

9. Голубев Ю.Ф., Мелкумова Е.В. Существование заданного движения робота при использовании трубы в качестве опоры. В сборнике VII Всероссийское совещание-семинар заведующих кафедрами и преподавателей теоретической механики, робототехники, мехатроники вузов Российской Федерации. Махачкала, 2016, С. 30-33.

10. Golubev Yu.F., Melkumova E.V. Two-legged Walking Robot Prescribed Motion On a Rough Cylinder // AIP Conference Proceedings, Vol. 1959, 2018, № 030009, pp. 030009-1-030009-8.

11. Yu.F. Golubev, Melkumova E.V. An Analogy of the Equilibrium of a Two-legged Robot on a Cylinder for the Problem of Transfer by a Manipulator With a Two-finger Grasp of a Cylinder, APM 2018, Proceedings of XLVI Summer School-Conf. "Advanced Problems in Mechanics", June 25-30, St. Petersburg, Russia; Polytech-IPME RAS, pp. 117-124.

12. Golubev Yu.F., Melkumova E.V. Footholds Admissible Areas Structure of a Two-legged Walking Robot on an Inclined Cylinder//Conference Series: Materials Science and Engineering, v. 468, № 012003, 2018, pp. 1-9. 
13. Golubev Yu.F., Melkumova E.V. Brittle Cylinder Transfer by a Three-finger Grasp//VIII International Conference on Computational Methods for Coupled Problems in Science and Engineering (COUPLED 2019), Sitges (Barcelona), Spain, Испания, 3-5 июня 2019, https://congress.cimne.com/coupled2019/fronta1/doc/EbookCoupled2019.pdf, pp. 164-175.

14. Golubev Yu.F., Melkumova E.V. Transfer by a Manipulator with a Three-Finger Grasp of a Brittle Cylinder//APM 2019 Book of Abstracts, XLVII International Conference "Advanced Problems in Mechanics", June 24-29, 2019, St. Petersburg, Russia, тезисы, с. 78.

15. Голубев Ю.Ф., Корянов В.В., Мелкумова Е.В. Переворачивание шагающего робота в рабочее состояние из аварийного положения “вверх ногами”//Препринты ИПМ им. М.В.Келдыша. 2019. № 48. 22 c. doi:10.20948/prepr-2019-48 URL: http://library.keldysh.ru/preprint.asp?id=2019-48

16. Голубев Ю. Ф., Мелкумова Е. В. Перенос хрупкого цилиндра трёхпальцевым манипулятором // XII Всероссийский съезд по фундаментальным проблемам теоретической и прикладной механики. Аннотации докладов. - РИЦ БашГУ Уфа, 2019. — С. 55-55.

Статья поступила в редакцию 30 октября 2019 г. 